\title{
Anisotropía en el comportamiento a tracción de la aleación Ti-6Al-2Sn-4Zr-6Mo
}

\author{
M. Corzo*, J.M. Manero*, M. Anglada* y A. Mateo*
}

\begin{abstract}
Resumen Se ha realizado un estudio del comportamiento mecánico a tracción de la aleación Ti-6Al2Sn-4Zr-6Mo, forjada en la región $\beta$ con un tratamiento posterior de doble envejecimiento. Puesto que esta aleación suele presentar una fuerte anisotropía, se consideraron las direcciones radial y tangencial con respecto al eje de forja. Se ha observado una fuerte disminución en el límite de elástico para la dirección radial junto con un notable aumento de la ductilidad. Análisis mediante microscopía electrónica de transmisión y difracción de rayos X, permiten concluir que este comportamiento está asociado con la transformación martensítica inducida por deformación, la existencia de maclado, y en menor grado con la presencia de fase $\alpha$, a lo largo de los límites de grano.
\end{abstract}

Palabras clave Ti-6246. Propiedades en tracción. Textura. Deformación.

\section{Anisotropy in the tensile behaviour of Ti-6Al-2Sn-4Zr-6Mo alloy}

\begin{abstract}
Tensile properties were evaluated for a Ti-6Al-2Sn-4Zr-6Mo alloy forged, in the $\beta$ state age-hardening state and further double ageing treatment. Since this alloy tends to exhibit a strong anisotropy in the mechanical behaviour, the study has been realized in the radial and tangential direction relative to the forging axis. An anomalous tendency was observed in the tensile test, characterised by low yield strength and a high ductility in the radial direction. This effect was analyzed by transmission electron microscopy and X-ray diffraction. The phase transformation to martensite induced by deformation, the mechanical deformation by twinning and the presence of a-phase along the grain boundary of the colonies (b-prior) are the reasons for this anomalous tendency.
\end{abstract}

Keywords

Ti-6246. Mechanical Properties. Texture. Deformation.

\section{INTRODUCCIÓN}

Los materiales actualmente usados por la industria aeronáutica para la elaboración del compresor en las turbinas a gas son, principalmente, aleaciones de titanio $(\alpha / \beta)$ y aleaciones base níquel. En el caso de las primeras, las microestructuras resultantes del conformado en la región $\beta$ y los posteriores envejecimientos a alta y baja temperatura llamadas $\beta$-processed, son la mejor vía para minimizar los inconvenientes que generan la presencia de $\alpha$ en los límites de grano de la fase $\beta$. Mediante este tratamiento se logra que las capas de fase $\alpha$ sean discontinuas en dichos límites de grano ${ }^{[1]}$, evitando, de esta manera, estas zonas preferenciales de deformación. Como resultado de los procesos de confor- mado y tratamiento térmico, estas aleaciones pueden adquirir textura cristalográfica ${ }^{[2]}$. En la literatura se encuentra referido un comportamiento anómalo a tracción en la aleación Ti-6246, caracterizado por oscilaciones en la curva esfuerzo- deformación a velocidades constantes de deformación, el cual se atribuye a una transformación martensítica inducida por deformación ${ }^{[3]}$. Es bien conocido que la fase $\beta$ metaestable, presente en aleaciones de titanio $(\alpha+\beta)$ y $\beta$, se puede transformar por deformación, en fase $\alpha^{\prime}$ (martensita hexagonal), $\alpha$ " (martensita ortorrómbica) o en fase $\omega$ (precipitados coherentes-hexagonales). Estas transformaciones afectan a la ductilidad y a la tenacidad de la aleación. Asimismo, la fase $\alpha$ " (martensita ortorrómbica) también se puede formar por medio

(*) Departamento de Ciencia de Materiales e Ingeniería Metalúrgica, Universitat Politècnica de Catalunya. Avda. Diagonal 647, Barcelona. 
de una transformación metaestable durante el temple $^{[4]}$.

El presente trabajo se centra en estudiar la influencia de la textura y de la transformación martensítica inducida por deformación en el comportamiento a tracción de la aleación Ti-6Al-2Sn4Zr-6Mo. Para ello, se han llevado a cabo ensayos de tracción en las direcciones radial y tangencial con respecto al eje de forja.

\section{MATERIALES Y PROCEDIMIENTO EXPERIMEN- TAL}

La aleación estudiada Ti-6Al-2Sn-4Zr-6Mo, generalmente designada como $\mathrm{Ti}-6246$, fue colada en forma de disco $(\varnothing 400 \mathrm{~mm} \times 50 \mathrm{~mm})$. La forja se realizó en la región $\beta$ ( $\beta$-transus $945^{\circ} \mathrm{C}$ ). A continuación se sometió a un envejecimiento a $900{ }^{\circ} \mathrm{C}$ durante $1 \mathrm{~h}$, enfriándose a una velocidad controlada de $50^{\circ} \mathrm{C} / \mathrm{min}$ y, por último, se efectuó un segundo envejecimiento a $585^{\circ} \mathrm{C}$ durante $8 \mathrm{~h}$, seguido de enfriamiento al aire.

En la figura 1 puede observarse la microestructura de la aleación caracterizada mediante microscopía electrónica de barrido (MEB). Como resultado de la forja en la región $\beta$, los granos no recristalizados de fase $\beta$ tienen forma de pancake, obteniendo, así, una estructura de granos de aproximadamente $1,5 \mathrm{~mm}$ de largo, con capas de fase $\alpha$ discontinuas a lo largo de los límites de grano. (Fig. 1a)). En el enfriamiento desde la región $\beta$, se forman agujas de $\alpha$ dentro de los granos de $\beta$. Dichas agujas continúan creciendo durante el envejecimiento a alta temperatura. A su vez, se tienen placas de fase $\alpha$, llamadas "side plates", las cuales nuclean en el límite de grano de $\beta$ y crecen dentro de las colonias, siguiendo una orientación en zigzag. Durante el envejecimiento a baja temperatura, precipita una gran cantidad de placas finas de $\alpha$ secundaria, las cuales aparecen señaladas con una la flecha en la micrografía de la figura 1b). Debido a su reducido tamaño, estas placas han sido vistas más claramente por microscopía electrónica de transmisión (MET).

Para estudiar la variación de las propiedades mecánicas en función de la dirección con respecto al eje de forja, se llevaron a cabo ensayos de tracción, a una velocidad de desplazamiento constante de $2 \mathrm{~mm} / \mathrm{min}$, en probetas planas. De la zona deformada de las probetas ensayadas se extrajeron láminas delgadas para MET. Se utilizó la técnica de difracción de rayos-X para determinar la presencia de fase $\alpha$ ".
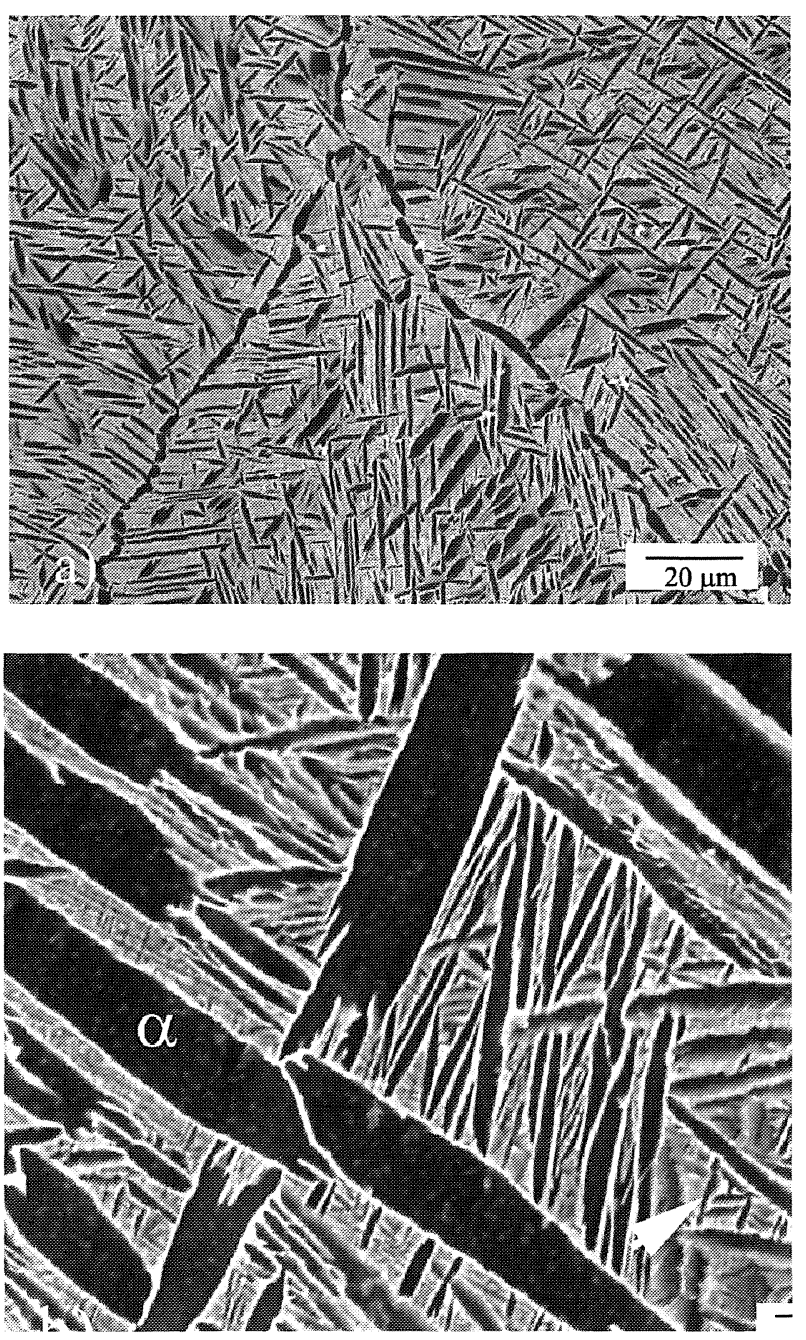

Figura 1. Microestructura de la aleación Ti-6246. a) Metalografía mediante MEB. b) Detalle de la fase $\beta$.

Figure 1. Ti-6246 microstructure. a) SEM micrograph. b) Detail of $\beta$ phase.

\section{RESULTADOS Y DISCUSIÓN}

Las dos orientaciones estudiadas presentan un comportamiento a tracción diferente en la curva esfuerzo-deformación (Fig. 2). El límite elástico obtenido en la dirección $\mathrm{T}$ es de $1.073 \mathrm{MPa}$; sin embargo, en la dirección $\mathrm{R}$, se reduce más de un 50 $\%$; la ductilidad evaluada mediante la reducción de área (RA) es menor en la dirección $\mathrm{T}$, como lo muestran los valores obtenidos para los diferentes ensayos (Tabla I).

En general, la ductilidad en tracción está principalmente determinada por dos parámetros: la resistencia a la nucleación de grietas, considerado el parámetro más influyente; pero también la resistencia a la propagación tiene cierta influencia ${ }^{[5]}$. En el caso de las aleaciones de titanio hay que destacar 


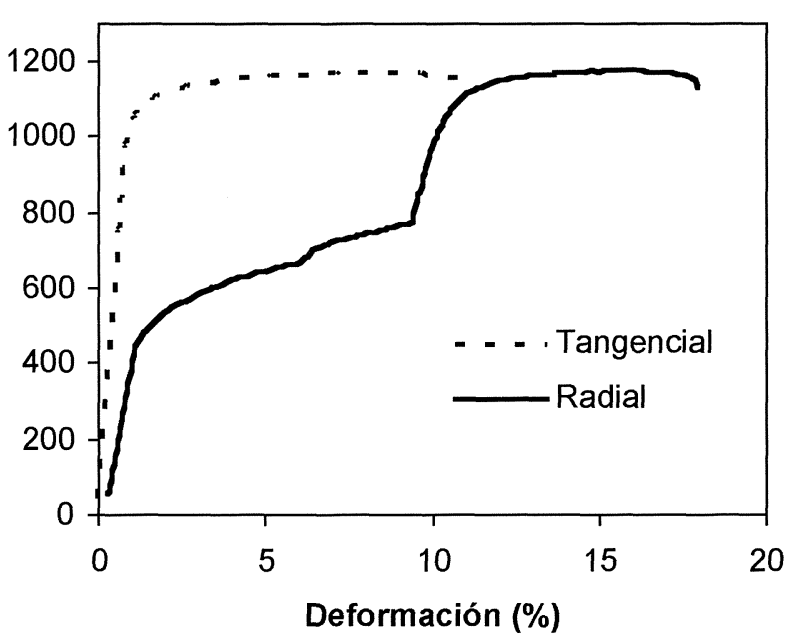

Figura 2. Comparación de los resultados obtenidos en los ensayos de tracción.

Figure 2. Stress-Strain plot showing test for different orientations.

Tabla I. Propiedades a tracción

Table I. Tensile properties

\begin{tabular}{lccc}
\hline Orient. & $\sigma_{0,2}(\mathrm{MPa})$ & $\sigma_{\text {UTS }}(\mathrm{MPa})$ & $\mathrm{RA}(\%)$ \\
\hline $\mathrm{T}$ & 1073 & 1086 & 16 \\
$\mathrm{R}$ & 480 & 1100 & 20 \\
\hline
\end{tabular}

la influencia de la fase $\alpha$ como zona preferencial de deformación. La buena ductilidad obtenida en la aleación de titanio se debe a la discontinuidad de las capas de $\alpha$ presentes en los límites de grano.

En las superficies de fractura se distinguen los granos de la fase $\alpha$ y $\beta$ (Fig. 3b) y 3c)). A su vez, hay un crecimiento de grieta intergranular (límite de grano $\beta$ previo) con porciones de fractura dúctil en la fase $\beta$, marcando de forma muy clara la estructura de agujas presente en el interior de las colonias. También se puede ver descohesión en los granos de la fase alfa, como lo muestra la figura 3a).

Para poder explicar el comportamiento a tracción en la dirección $\mathrm{R}$, se deben discutir dos aspectos: por una parte, la dependencia del límite elástico con la orientación está, probablemente, asociada con la textura cristalográfica presente, ya que es bien conocido en las aleaciones de titanio que la orientación del plano basal de la fase $\alpha$ con respecto a la dirección de aplicación de la carga ejerce una marcada influencia sobre las propiedades mecánicas ${ }^{[6]}$. Por ejemplo, una variación en el límite elástico, en función de la orientación de colonias de $\alpha$ y $\beta$ de la aleación Ti-1-1-8-1, ha sido
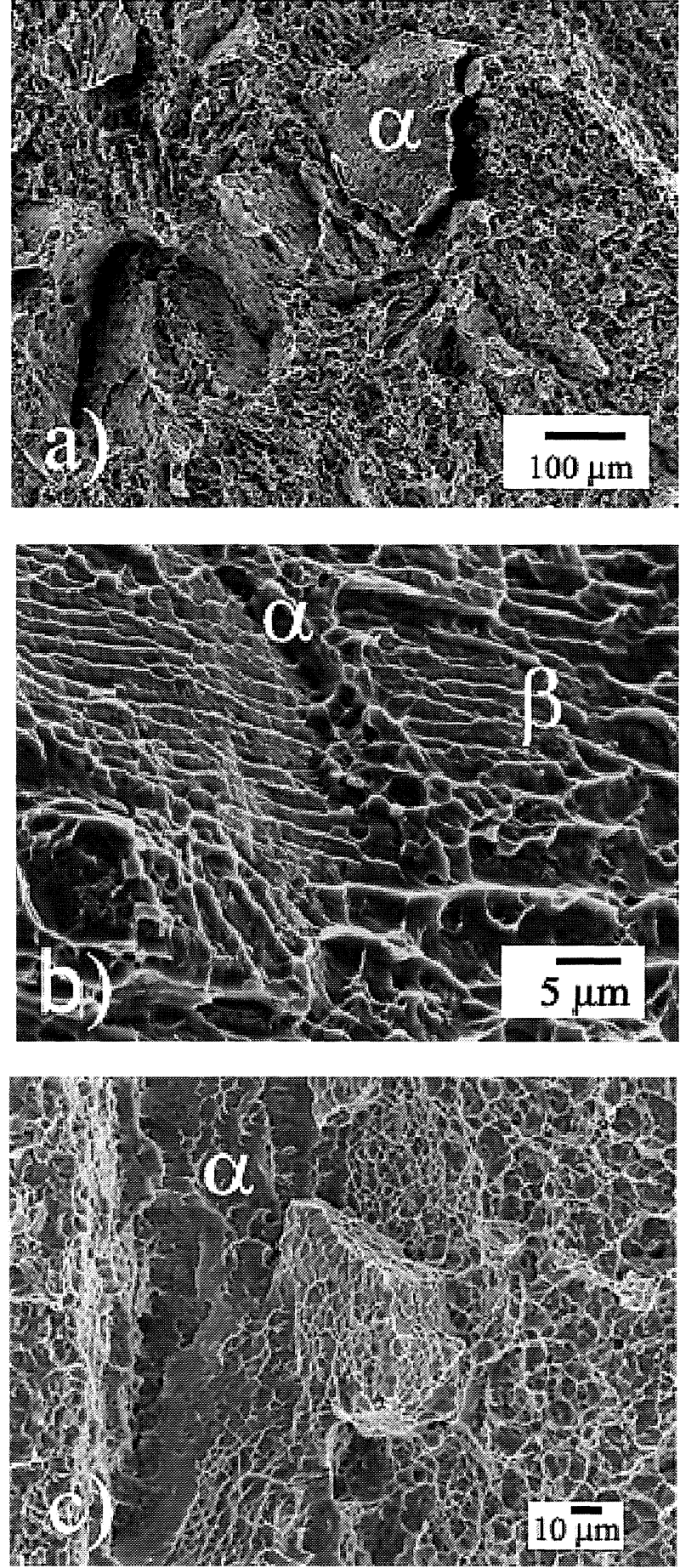

Figura 3. Micrografías de las superficies de fractura. a) Probeta tangencial. b) y c) Probeta radial.

Figure 3. Fracture surfaces. a) Tangencial specimens. b) and c) Radial specimens.

reportada por Chan, et al. ${ }^{[7]}$, quienes encontraron un aumento en el límite elástico para colonias cuya dirección de deslizamiento era casi normal a la interfase $\alpha / \beta$. Inclusive, la morfología de la fase $\alpha$ influye en los sistemas de deslizamiento que se puedan activar ${ }^{[8]}$. Por otra parte, S. Suri et al. ${ }^{[9]}$, 
consideran que el origen de esta anisotropía se debe al relativo desalineamiento de los sistemas de deslizamiento entre la fase $\alpha$ y $\beta$.

La forma de la curva esfuerzo-deformación correspondiente a la orientación $\mathrm{R}$ también puede estar relacionada con la transformación de $\beta$ a $\alpha$ " inducida por deformación. De acuerdo con la bibliografía ${ }^{[10]}$, esta transformación ocurre a un esfuerzo mínimo de $150 \mathrm{MPa}$. Nótese que, en el presente caso, la transformación se inicia aproximadamente a $450 \mathrm{MPa}$, lo cual se debe al aumento de la estabilidad de la fase $\beta$ debido a la presencia de elementos $\beta$ estabilizantes (Mo y V) ${ }^{[11]}$. Para verificar esta posibilidad se procedió a evaluar mediante MET y difracción de rayos X las características de las probetas antes y después de los ensayos de tracción.

La fase $\alpha$ " presenta una orientación cristalográfica aproximada con la fase $\beta$ original de: $(110)_{\beta} / /(002)_{\alpha \text { " y }}[111]_{\beta} / /[110]_{\alpha \text { ". }}$. Por otra parte, la transformación de $\beta$ - $\alpha$ " se favorece por el maclado de los planos $\{111\}_{\alpha^{\prime \prime \prime}}$. De lo anterior, y observando el patrón de difracción obtenido para la probeta radial después del ensayo de tracción (Fig. 4a), se puede constatar que la matriz de fase $\beta$ correspondiente al eje de zona $[111]_{\beta}$ presenta $\alpha$ " maclada con un plano de hábito (111) $)^{\prime \prime}$ (Fig. 4b), corroborando la transformación de fase inducida por deformación en la dirección radial.
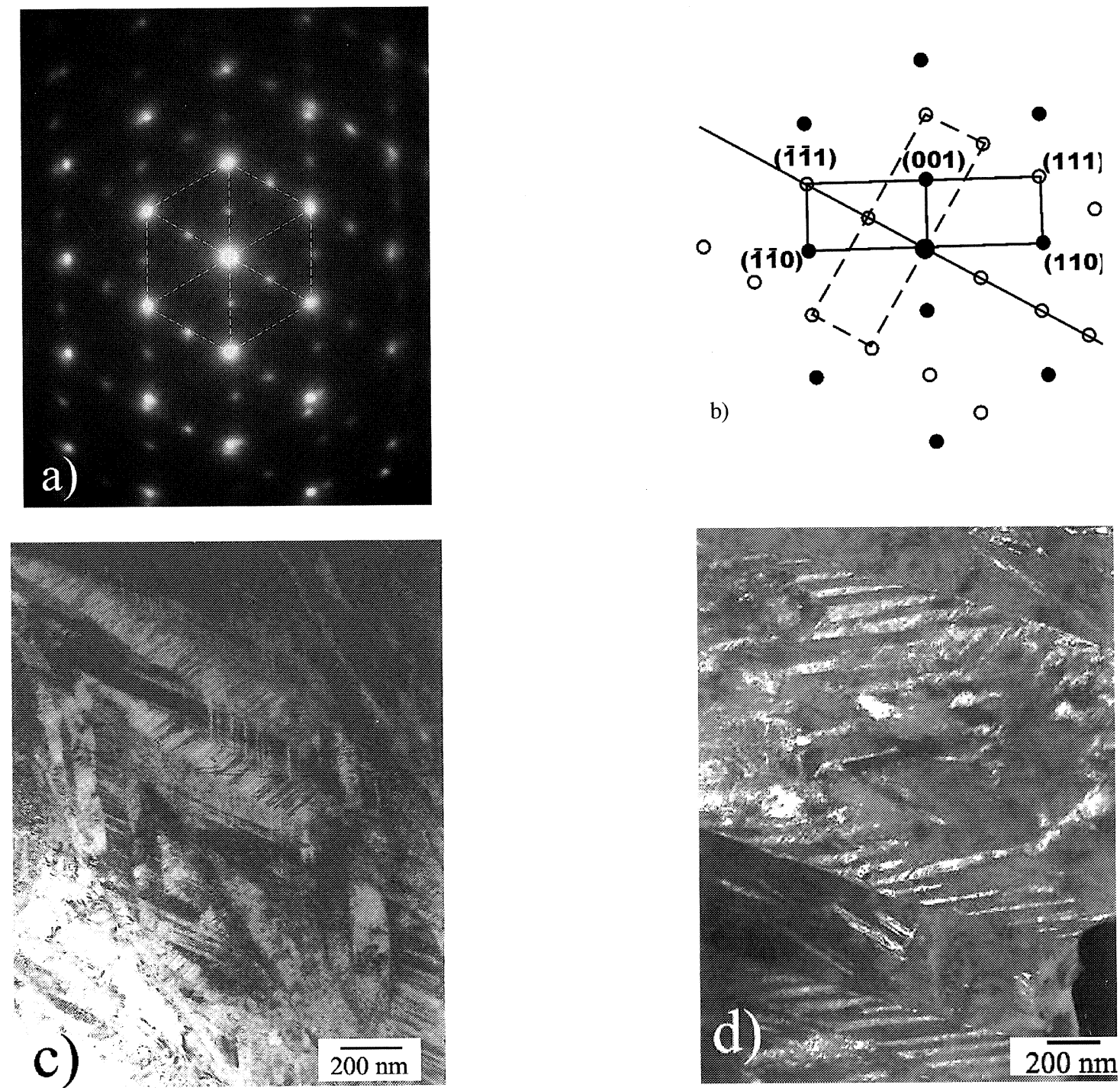

Figura 4. Micrografías de MET correspondientes a la dirección radial después del ensayo. a) Patrón de difracción de una placa de $\beta$ con placas de martensita ortorrómbica $\alpha^{\prime \prime}$, eje de zona $[111]_{\beta}||[10]_{\alpha^{\prime \prime}}$. b) Esquema indexado de $\alpha^{\prime \prime}$ y su macla. c) Micrografía en campo claro de martensita $\alpha^{\prime \prime}$ en $\beta$, eje de zona [1 111$] \beta$. d) Detalle micrografía anterior en campo oscuro.

Figure 4. TEM micrographs of radial section after tensile test. a) Patterns from $\beta$ layer with stress induced martensite $\alpha^{\prime \prime}$ from the $[111]_{\beta} \|[10]_{\alpha^{\prime \prime}}$ zone axes. b) Schematic diffraction of twin pattern of $\alpha^{\prime \prime}$. c) Bright-field TEM image of $\beta$ with stress induced martensite $\alpha^{\prime \prime}$. d) Dark-field TEM image. 
La presencia de martensita ortorrómbica $\alpha$ " también se constató mediante los estudios de difracción de rayos X. De forma cualitativa, se observa un desdoblamiento en los picos correspondientes al plano basal $(0002)_{\alpha}$, y $(110)_{\beta}$ (Fig. 5a), debido a un solapamiento con los picos (002) y (111) de $\alpha$ ", que, de acuerdo a lo referenciado en la literatura ${ }^{[6]}$, aparecen aproximadamente en los ángulos $2 \theta, 38,3$ y 39,7 , respectivamente.

En la micrografía de la figura 4c) se aprecia que la fase $\alpha$ exhibe maclado. Este mecanismo también contribuye a la deformación plástica de la fase $\alpha$, y es bien conocido que ocurre en una variedad de planos piramidales como son $\{10 \overline{1} 1\}$, $\{10 \overline{1} 2\},\{11 \overline{2} 1\},\{11 \overline{2} 2\},\{11 \overline{2} 3\}$ y el $\{11 \overline{2} 4\}^{[12]}$.
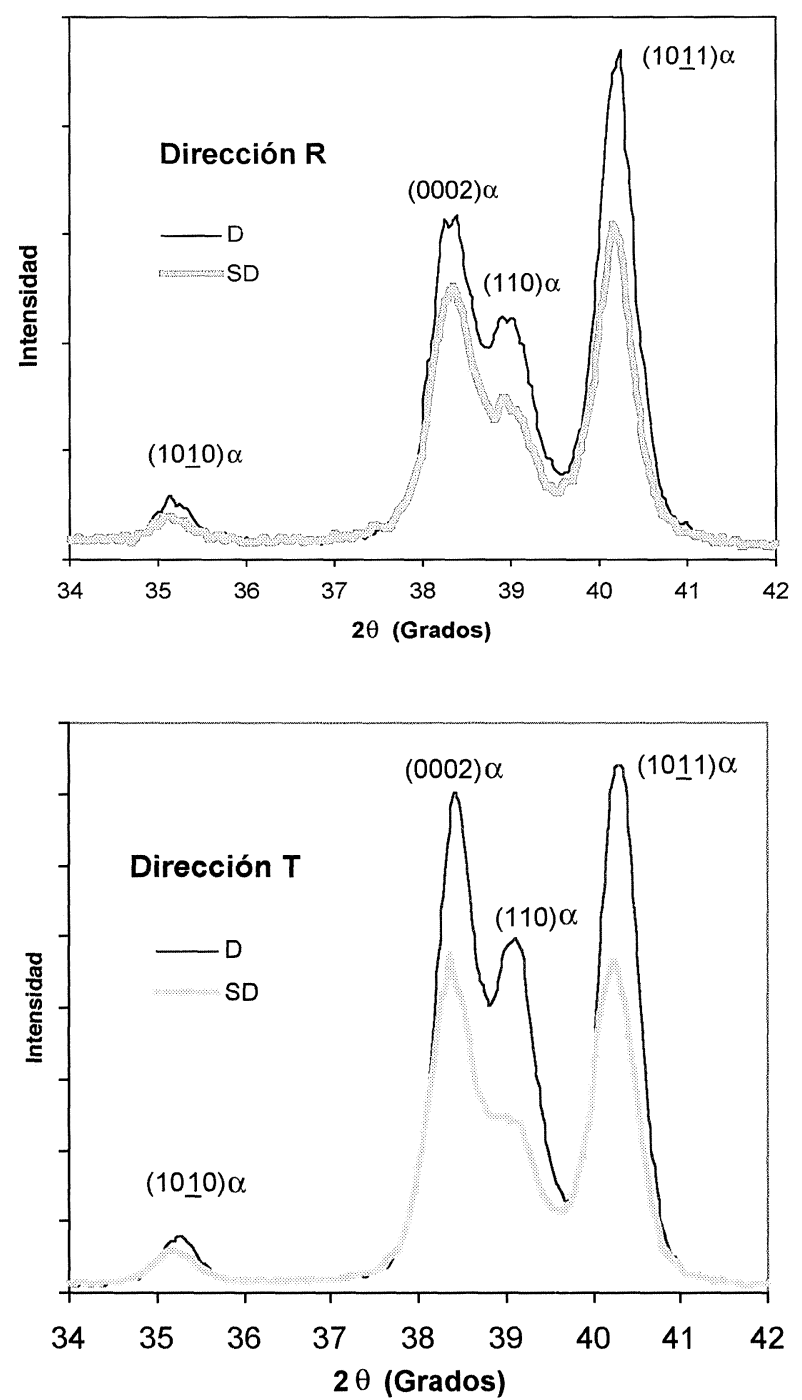

Figura 5. Difractogramas de rayos $X$ comparando la condición $\mathrm{D}$ (deformada) y SD (sin deformar) para cada una de las orientaciones $R$ y $T$ a) $R, b$ ) $T$.

Figure 5. X-ray diffraction profiles of deformed (D) and undeformed $(S D)$ specimens.
La técnica de difracción de rayos X no permitió detectar la presencia de martensita ortorrómbica para la dirección tangencial. Por otra parte, las variaciones de intensidad de los picos que corresponden a las muestras deformadas (D) con respecto a las sin deformar (SD), se deben a la textura propia del material, ya que la intensidad estará condicionada a las características de la zona evaluada.

\section{CONCLUSIONES}

El análisis microestructural de la aleación Ti-6Al$2 \mathrm{Sn}-4 \mathrm{Zr}$-6Mo muestra que las altas temperaturas asociadas a los procesos de forja en la región $\beta$ de aleaciones de titanio $\alpha+\beta$ dan como resultado un gran tamaño de grano de $\beta$ y una estructura Widmanstätten bastante gruesa, con una fase $\alpha$ alineada o con textura localizada. Sin embargo, con los tratamientos térmicos posteriores se provoca la formación de un entramado de fase $\alpha$, el cual mejora el comportamiento mecánico de la aleación.

Los resultados obtenidos en este estudio, indican que las probetas mecanizadas en el sentido radial con respecto al eje de forja presentan un menor límite elástico y una mayor ductilidad que las correspondientes al sentido tangencial. Análisis por difracción de rayos $\mathrm{X}$ y de electrones confirman la presencia de martensita ortorrómbica $\alpha$ " inducida por deformación en la dirección radial, por lo que el comportamiento observado se atribuye a esta transformación.

En las muestras tangenciales, no se detectó la presencia de $\alpha$ ", debido posiblemente a que existen orientaciones preferenciales que favorecen la transformación.

\section{Agradecimientos}

Los autores agradecen la financiación de este trabajo por la CICYT (Proyecto MAT2002-00368) y a la empresa MTU Aero Engines y al Dr. Olaf Roder por el suministro del material que ha sido utilizado en este proyecto.

\section{REFERENCIAS}

[1] C. Sauer y G. Lütjering, J. Mater. Process. Tech. 117 (2001) 311-317.

[2] H.J. Bunge, In H. Bunge (Ed.), Directional Properties of Materials, 1998, pp. 1-7.

[3] M. Ninomi, T. Kobayaskhi, I. Inagaki y A.W. Thompson, Met. Trans. A 21 (1990) 1.773-1.744. 
[4] M. Young, E. Levine y H. Margolin, Met. Trans. 5 (1974) 1.891-1.898.

[5] C. Sauer y G. Lütjering, Mat. Sci. Eng. A 319-321 (2001) 393-397.

[6] M.R. Bache y W.J. Evans, Mat. Sci. Eng. A A319-321 (2001) 409-414.

[7] K.S. Chan, C.C. Wojcik y D.A. Koss, Met. Trans. A 12 (1981) 1.899.

[8] A. Ambard, L. GuétAz, F. Louchet y D. Guichard, Mat. Sci Eng. A 319-321 (2001) 404-408.
[9] S. Suri, G.B. Viswanathan, T. NeERAJ, D.H Hou y M.J. Mills, Acta Mater. 47 (1999) 1.019-1.034.

[10] Materials Properties Handbook ASM, Titanium Alloys, 1994, pp. 1.051-1.078

[11] T.W. Duering, G.T. Terlinde y J.C. Williams, Met. Trans. A 11 (1980) 1.987-1.998.

[12] J.M. Manero, F.X. Gil y J.A. Planell, Tinanium'99: Science and Technology, 1999, pp. 458-465. 